\title{
Re: Risk of pre-eclampsia after gastric bypass: a matched cohort study
}

\author{
Marleen van Gelder ${ }^{1}$, Hubertina Scheepers ${ }^{2}$, and Luc Smits ${ }^{3}$ \\ ${ }^{1}$ Radboudumc \\ ${ }^{2}$ Maastricht UMC+ \\ ${ }^{3}$ Maastricht University
}

September 26, 2021

\section{BJOG Exchange}

\section{Re: Risk of pre-eclampsia after gastric bypass: a matched cohort study}

\author{
Marleen M.H.J. van Gelder, ${ }^{1}$ Hubertina C.J. Scheepers, ${ }^{2}$ Luc J.M. Smits ${ }^{3}$
}

${ }^{1}$ Department for Health Evidence, Radboud Institute for Health Sciences, Radboud university medical center, P.O. Box 9101, 6500 HB Nijmegen, The Netherlands.

${ }^{2}$ Department of Obstetrics and Gynaecology, Maastricht University Medical Centre, P.O. Box 5800, 6202 AZ Maastricht, The Netherlands.

${ }^{3}$ Department of Epidemiology, CAPHRI Care and Public Health Research Institute, Maastricht University, P.O. Box 616, 6200 MD Maastricht, The Netherlands.

Corresponding author:

Dr. Marleen van Gelder, Department for Health Evidence (HP 133), Radboud university medical center, P.O. Box 9101, 6500 HB Nijmegen, The Netherlands. Tel: +31-24-3666126. Email: Marleen.vanGelder@radboudumc.nl.

Sir ,

We read with great interest the research article by Dr Johansson et al., ${ }^{1}$ in which Swedish nationwide healthcare data were used to assess the association between gastric bypass before pregnancy and the risk of pre-eclampsia. We applaud the authors for their careful matching approach in the selection of the control groups to prevent confounding. However, we feel that the conclusion that gastric bypass in itself is effective in the prevention of pre-eclampsia may still be considered premature.

Because of the higher risk of nutritional deficiencies due to micronutrient malabsorption after bypass procedures, lifelong micronutrient supplementation is started at hospital discharge after bariatric surgery. This recommendation includes supplementation of, among others, vitamin D (3000 International Units $\mathrm{D}_{3}$ daily from all sources) and calcium (1200-2400 mg daily in divided doses, depending on the type of bariatric procedure), ${ }^{2}$ both of which have shown to decrease the risk of pre-eclampsia. In a meta-analysis of 27 randomized controlled trials, vitamin D supplementation was found to lower the risk of pre-eclampsia with almost two-thirds (odds ratio $0.37,95 \%$ confidence interval $0.26-0.52) .{ }^{3}$ A reduced risk was also observed after high-dose calcium supplementation ([?]1 g/day) in a meta-analysis of 13 trials (relative risk $0.45,95 \%$ confidence interval 0.31-0.65). ${ }^{4}$ Although vitamin D increases intestinal calcium absorption, co-administration 
of vitamin $\mathrm{D}$ and calcium does not seem to result in additional benefit. ${ }^{3}$ Several biological pathways for protective effects of vitamin $\mathrm{D}$ and calcium on the occurrence of pre-eclampsia have been proposed..$^{3,4}$

Johansson et al. used propensity score matching to adjust for multiple potential confounders, including pre-surgery or early-pregnancy BMI, pre-existing diabetes, maternal age, smoking status, educational level, height, country of birth, delivery year, and history of pre-eclampsia. ${ }^{1}$ However, micronutrient supplementation was not taken into account in the analyses, whereas at least a substantial part of the reduction in the risk of pre-eclampsia observed after gastric bypass before pregnancy could be attributable to supplementation of vitamin $\mathrm{D}$ and calcium. With the current study design, it is challenging to distinguish between the effect of gastric bypass itself and the effect of vitamin $\mathrm{D}$ and calcium supplementation that was initiated directly after the procedure on the risk of pre-eclampsia, as virtually all women with a history of gastric bypass will have used supplements during pregnancy. Although all pregnant women are routinely counselled on vitamin D and calcium supplementation, we know from clinical practice that uptake of these recommendations is low. Restricting the matched control groups to women who also used vitamin D and calcium supplements with similar dosages during pregnancy may solve this problem, but it is questionable whether reliable information on supplement use is available from nationwide registries.

Disclosures of interests:

The authors have no conflicts of interest to report.

Contribution to authorship:

MvG conceived and drafted the letter. HS and LS contributed revisions and significant intellectual content. All authors approved of the final version for publication.

Funding:

MvG was supported by the Netherlands Organisation for Health Research and Development [ZonMw; grant number 848018010].

References:

1. Johansson K, Wikström AK, Söderling J, Näslund I, Ottoson K, Neovius M, et al. Risk of pre-eclampsia after gastric bypass: a matched cohort study. BJOG 2021; https://doi.org/10.1111/1471-0528.16871.

2. Mechanick JI, Apovian C, Brethauer S, Garvey WT, Joffe AM, Kim J, et al. Clinical practice guidelines for the perioperative nutrition, metabolic, and nonsurgical support of patients undergoing bariatric procedures - 2019 update: Cosponsored by American Association of Clinical Endocrinologists/American College of Endocrinology, The Obesity Society, American Society for Metabolic and Bariatric Surgery, Obesity Medicine Association, and American Society of Anesthesiologists. Obesity 2020;28:O1-O58.

3. Fogacci S, Fogacci F, Banach M, Michos ED, Hernandez AV, Lip GYH, et al. Vitamin D supplementation and incident preeclampsia: a systematic review and meta-analysis of randomized clinical trials. Clin Nutr 2020;39:1754-1752.

4. Hofmeyr GJ, Lawrie TA, Atallah ÁN, Torloni MR. Calcium supplementation during pregnancy for preventing hypertensive disorders and related problems. Cochrane Database Syst Rev 2018;10:CD001059. 